\title{
Frecuencia de dermatofitosis en bovinos Bos indicus del departamento de Córdoba, Colombia
}

\author{
FREQUenCy OF DERMATOPHYTOSIS IN BOVINe (Bos indicus) OF CóRdoba \\ DEPARTMENT, COLOMBIA
}

\author{
José Cardona-Álvarez ${ }^{1,3}$, Donicer E. Montes-Vergara ${ }^{2}$, Nicolás Martínez-Humanes ${ }^{1}$
}

\section{Resumen}

Se realizó un estudio retrospectivo de la dermatofitosis cutánea bovina a través de los registros clínicos entre junio de 2007 y junio de 2017 del Servicio Clínico Ambulatorio de Grandes Animales de la Facultad de Medicina Veterinaria de la Universidad de Córdoba, Colombia. El objetivo fue determinar la frecuencia de dermatofitosis cutánea que ocurren en bovinos (Bos indicus) en el Departamento de Córdoba. De los casos dermatológicos atendidos en ese periodo (1184), $253(21.4 \%)$ correspondieron a dermatofitosis cutánea en bovinos de explotaciones ganaderas. El 54.6\% fueron machos. El grupo etario más afectado fue el menor de 12 meses con el 56.2\% (142/253). Las ubicaciones de lesiones más comunes fueron a nivel de cuerpo (tórax y abdomen) (39.9\%), cuello $(27.3 \%)$ y cabeza $(24.5 \%)$. No hubo diferencia significativa en la frecuencia de dermatofitosis con respecto a la edad, sexo y ubicación de las lesiones.

Palabras clave: enfermedades de piel; dermatomicosis; dermopatología

\section{Abstract}

A retrospective study of bovine dermatophytosis was carried out through the clinical records between June 2007 and June 2017 of the Ambulatory Clinical Service of Large Animals of the Faculty of Veterinary Medicine of the University of Córdoba, Colombia. The aim was to determine the frequency of cutaneous dermatophytosis that occur in bovines (Bos indicus) in the Department of Córdoba. Of the 1184 dermatological cases

\footnotetext{
${ }^{1}$ Facultad de Medicina Veterinaria y Zootecnia, Universidad de Córdoba, Montería, Colombia

${ }^{2}$ Facultad de Ciencias Agropecuarias, Universidad de Sucre, Sincelejo, Colombia

${ }^{3}$ E mail: jacardonaalvarez@correo.unicordoba.edu.co
}

Recibido: 30 de octubre de 2017

Aceptado para publicación: 10 de mayo de 2018 
attended in that period, $253(21.4 \%)$ corresponded to cutaneous dermatophytosis in cattle. Of them, $54.6 \%$ were males. The most affected age group was the younger than 12 months with $56.2 \%(142 / 253)$. The location of the most common lesions was at the level of the body (chest and abdomen) (39.9\%), neck $(27.3 \%)$ and head (24.5\%). There was no significant difference in the frequency of dermatophytosis with respect to age, sex and location of lesions.

Key words: skin disease; dermatomycosis; dermopathology

\section{INTRODUCCIÓN}

La dermatofitosis cutánea bovina (DCB), tambien conocida como dermatomicosis, flavus, herpes o tiñas (Rodríguez et al., 2002), pertenece a un grupo de enfermedades contagiosas de distribución mundial producidas por hongos que provocan lesiones en la piel, pelos y tegumentos cornificados (Chermette et al., 2008).

Es causado por dermatofitos de los géneros Trichophyton, Microsporum y Epidermophytum; de los cuales las especies Trichophyton verrucosum y, en menor medida, Trichophyton mentagorphyton, son las responsables de causar la enfermedad en el bovino (Avante et al., 2009; Antúnez et al., 2012).

Las lesiones características en los bovinos consisten en la presencia de placas circulares, blancas o grisáceas, secas y bien delimitadas, pudiendo llegar a desarrollar una infección bacteriana secundaria a causa del rascado intenso (Antúnez et al., 2014). Se distribuyen a nivel de cabeza y cuello y, en menor proporción, a nivel de miembros y región inguinal (Rodríguez et al., 2002).

La transmisión se da principalmente en actividades grupales por contacto directo con animales enfermos o a través de corrales o materiales de trabajo como sogas o herramientas contaminadas (Antúnez et al., 2014; Avante et al., 2009). El estado inmunológico del animal es determinante, ya que factores como la desnutrición, aplicación de medicamentos inmunosupresivos, confinamiento y estrés son determinantes para la presentación de la dermatofitosis (Antúnez et al., 2014).

El diagnóstico se basa en la caracterización clínica de las lesiones, las cuales son circunscritas, costrosas y fácilmente sangrantes (Cardona, 2016). Así mismo, se describen lesiones clásicas como pequeñas costras en la base de mechones de pelos, que posteriormente caen formando un área con alopecia focal o diseminada, descamativa, con ligero eritema y de evolución centrífuga lenta, de 1-8 $\mathrm{cm}$ de diámetro, pudiendo presentar prurito (Chermette et al., 2008; Cornegliani et al., 2009). Para el examen microscópico del raspado de las lesiones se clarifica las muestras con hidróxido de potasio $(\mathrm{KOH})$ al $10-20 \%$ y se observan a $10 \mathrm{X}$ y $40 \mathrm{X}$, para determinar la presencia de hifas artroconidiadas que confirman el diagnóstico, el cual, mediante el tamaño, tipo de crecimiento y localización endotrix o ectotrix permite llegar el género (Reis-Gomes et al., 2012). El tratamiento es de tipo topical con soluciones de yodo o sistémico en caso de lesiones generalizadas con griseofulvina (50 $\mathrm{mg} / \mathrm{kg}$ ) o itraconazol $(5-10 \mathrm{mg} / \mathrm{kg}$ ) (Avante et al., 2009).

El objetivo del presente estudio fue determinar la frecuencia de presentación de dermatofitosis cutánea en bovinos Bos indicus del departamento de Córdoba, Colombia. 


\section{Materiales y Métodos}

Se realizó un estudio de tipo descriptivo retrospectivo en bovinos con dermatofitosis cutánea (DCB) diagnosticados a través del examen clínico y laboratorial del raspado cutáneo. El estudio se llevó a cabo en el departamento de Córdoba, Colombia, ubicado a una altura de $30 \mathrm{msnm}$, con $28^{\circ} \mathrm{C}$ de temperatura promedio anual, $82 \%$ de humedad relativa, $1400 \mathrm{~mm}$ de precipitación media anual, cuya formación climática corresponde a bosque tropical lluvioso (Pabón et al., 2001).

De un total de 1184 casos dermatológicos atendidos entre junio de 2007 y junio de 2017, 253 (21.3\%) correspondieron a casos clínicos de dermatofitosis cutánea en bovinos Bos indicus de explotaciones ganaderas del departamento de Córdoba, diagnosticados en el Servicio Clínico Ambulatorio de Grandes Animales de la Facultad de Medicina Veterinaria de la Universidad de Córdoba (Unicordoba). Los animales fueron de ambos sexos (138 machos y 115 hembras), con edades entre 3 y 36 meses y clasificados en tres grupos etarios $(<12,12-24,>24$ meses $)$.

Para la evaluación de los animales y la toma de muestras, se tuvieron en cuenta las normas técnicas referentes a los principios éticos internacionales para la investigación biomédica con animales del CIOMS (Council for International Organizations of Medical Sciences) establecida por la UNESCO (United Nations Educational, Scientific and Cultural Organisation) y la OMS (Organización Mundial de la Salud) de 1949 y de la Ley 84 del 27 de octubre de 1989 (Estatuto Colombiano de Protección Animal) (Mrad, 2006).

El diagnóstico de dermatofitosis fue realizado mediante examen clínico general y dermatológico especial (inspección directa, inspección con lupa, palpación y escarificación), raspado cutáneo, prueba de $\mathrm{KOH}$ y respuesta terapéutica. A todas las lesiones cutáneas se les realizó caracterización anatomopatológica, siendo tomadas fotografías de alta definición para su posterior análisis (Sony DSC-HX10V, China). Se realizaron raspados cutáneos superficiales y profundos en la periferia de las lesiones (Silveira et al., 2003), que posteriormente fueron procesados con la prueba de $\mathrm{KOH}$ en el laboratorio antes mencionado.

Se utilizó la prueba de Chi-cuadrado con el fin de determinar diferencias $(p<0.05)$ entre las variables (diagnóstico, sexo y edad). La tabulación, organización y el análisis estadístico de los datos fue realizado con el auxilio del programa de computador SAS v. 9.1.3.

\section{Resultados y Discusión}

Las manifestaciones clínicas de las lesiones observadas en los animales con DCB y los resultados de los raspados cutáneos confirmaron el diagnóstico de DCB en los 253 bovinos estudiados, de acuerdo con lo reportado como método de diagnóstico de la dermatofitosis cutánea en bovinos (Silveira et al., 2003; Chermette et al., 2008; Betancourt et al., 2009; Cornegliani et al., 2009; Reis-Gomes et al., 2012; Cardona, 2016). La frecuencia de presentación fue de $21.3 \%$ (253/1184). En varias explotaciones ganaderas se detectaron brotes de la enfermedad en grupos de diferentes edades o etapas productivas (Figura 1), evidenciando la fácil transmisión de la enfermedad (Antúnez et al., 2014).

En el Cuadro 1 se describe la distribución de la dermatofitosis cutánea según el sexo y edad de los animales y la ubicación de las lesiones. El grupo etario más afectado fue el menor de 12 meses con el 56.2\% (142/ 253), seguido del grupo de 12-24 meses con $30.0 \%(76 / 253)$. La ubicación de las lesiones más frecuentes fueron a nivel de tórax y abdomen $(39.9 \%)$, cuello $(27.3 \%)$ y cabeza 
Cuadro 1. Distribución de lesiones en 123 animales causadas por dermatofitosis cutánea bovina en Córdoba, Colombia, según sexo y edad (en meses)

\begin{tabular}{lcccccccc}
\hline $\begin{array}{l}\text { Ubicación de } \\
\text { las lesiones }\end{array}$ & \multicolumn{3}{c}{$\begin{array}{c}\text { Machos } \\
(\mathrm{n}=138)\end{array}$} & \multicolumn{3}{c}{$\begin{array}{c}\text { Hembras } \\
(\mathrm{n}=115)\end{array}$} & \multicolumn{2}{c}{ Total } \\
\cline { 2 - 10 } & $<12$ & $12-24$ & $>24$ & $<12$ & $12-24$ & $>24$ & $\mathrm{n}$ & $\%$ \\
\hline Cabeza & 18 & 8 & 4 & 20 & 7 & 5 & 62 & 24.5 \\
$\begin{array}{l}\text { Cuello } \\
\begin{array}{l}\text { Cuerpo } \\
\text { (tórax y }\end{array}\end{array}$ & 21 & 13 & 3 & 18 & 9 & 5 & 69 & 27.3 \\
$\begin{array}{l}\text { abdomen) } \\
\text { Extremidades }\end{array}$ & 7 & 15 & 8 & 25 & 19 & 7 & 101 & 39.9 \\
\hline Total & 73 & 3 & 2 & 6 & 2 & 1 & 21 & 8.3 \\
\hline \multicolumn{1}{c}{$(\%)$} & $(28.9)$ & $(15.4)$ & $(6.7)$ & $(27.3)$ & $(14.6)$ & $(7.1)$ & $(100)$ & \\
\hline
\end{tabular}

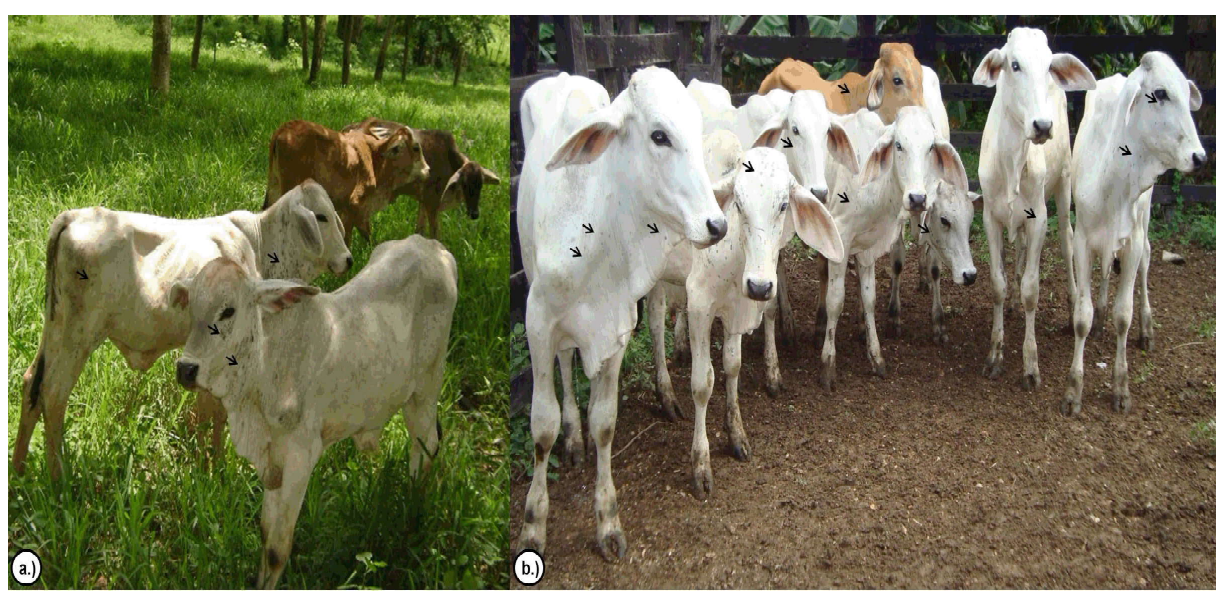

Figura 1. Lotes de bovinos afectados con dermatofitosis cutánea bovina. a.) Terneros lactantes menores de 12 meses. b.) Terneros de levante entre 12 y 24 meses

(24.5\%) (Figura 2). No hubo diferencia significativa en la frecuencia de dermatofitosis con respecto a la edad, sexo y ubicación de las lesiones en los animales del estudio.

En todas las muestras de raspados cutáneos y procesadas con $\mathrm{KOH}$ se observaron características similares, caracterizadas por presencia de estructuras fúngicas, tales como micelios artrosporados y hifas artroconidiadas (Silveira et al., 2003; Betancourt et al., 2009).
La frecuencia de presentación de dermatofitosis cutánea en bovinos fue del $21.3 \%$, situación que pudo ser favorecida por las condiciones agroecológicas de bosque tropical lluvioso, propicias para la presentación de enfermedades micóticas (Cardona et al., 2013). Los resultados concuerdas con lo reportado por Cardona et al. (2017) y Buitrago et al. (2017), quienes indican a la dermatofitosis como la segunda enfermedad dermatológica más frecuente en bovinos del departamento de Córdoba. Resultados infe- 


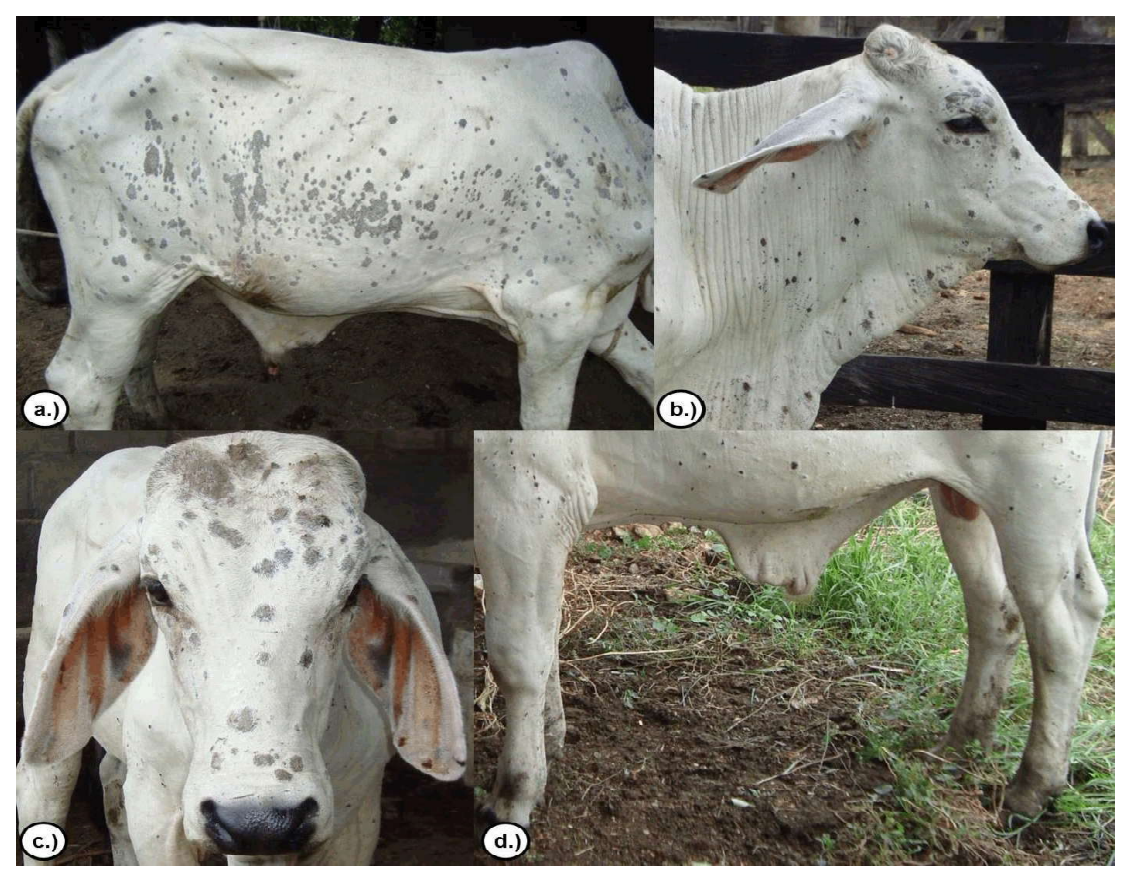

Figura 2. Ubicación cutánea de las lesiones micoticas a nivel de: a.) cuerpo (tórax y abdomen); b.) cuello; c.) cabeza; d.) extremidades

riores al del presente estudio fueron reportados por García et al. (2012) y Khosravi y Mahmoudi (2003) con prevalencias de 12.5 y $15 \%$ en bovinos de México e Irán, respectivamente. Asimismo, Pereira y Meireles (2007) reportaron brotes de dermatofitosis en bovinos con prevalencias entre 7.5 y $42.8 \%$. Por otro lado, Silveira et al. (2003) reportaron una prevalencia del $95.8 \%$ mediante examen directo con $\mathrm{KOH}$ en bovinos de Rio Grande del Sur, Brasil, mientras que Papini et al. (2009) y Agnetti et al. (2014) reportaron aislamientos de Trichophyton verrucosum en el 87.7 y $98.9 \%$, respectivamente, en animales sintomáticos, siendo el dermatófito más prevalente en todos los rebaños investigados.

Las lesiones se ubicaron con mayor presencia a nivel de tórax y abdomen, a diferencia de García et al. (2012), quienes encontraron mayor presencia de lesiones a nivel de cabeza y cuello, donde la transmisión de los hongos se efectúa fundamentalmente por contacto directo de animales enfermos con los sanos. De igual forma, Antúnez et al. (2014) expresan que la transmisión ocurre principalmente en actividades grupales donde hay mayor contacto o mediante materiales de trabajo como sogas o herramientas contaminadas.

Los animales afectados tenían entre 3 y 36 meses, habiendo mayor presentación en bovinos menores de 24 meses, coincidiendo con García et al. (2012). Es posible que los animales que enferman a edades tempranas alcancen un prolongado nivel de inmunidad y a que animales de mayor edad presentan un mayor grosor de piel, lo cual disminuye la receptividad al hongo. Así mismo, ReisGomes et al. (2012) expresan que las micosis afectan principalmente a menores de 12 meses, probablemente debido a que el sistema inmune no está totalmente activo; mientras que Avante et al. (2009) enfatizan en factores como la desnutrición, aplicación de medicamentos inmunosupresivos, confina- 
miento y estrés como aspectos determinantes para la presentación de dermatofitosis. Por otro lado, Papini et al. (2009) no encontraron diferencias con respecto a la edad y la presentación de dermatofitosis, y Agnetti et al. (2014) indicaron que la tiña típica estuvo presente en el $71.7 \%$ de los bovinos menores de 6 meses y solo en el $11 \%$ de los mayores de 6 meses.

Se concluye que las dermopatías micóticas son de alta presentación en bovinos del trópico bajo colombiano, por lo que se convierte en uno de los principales motivos de consulta veterinaria en el departamento de Córdoba.

\section{Literatura Citada}

1. Agnetti F, Righi C, Scoccia E, Felici A, Crotti S, Moretta I. Moretti A, et al. 2014. Trichophyton verrucosum infection in cattle farms of Umbria (Central Italy) and transmission to humans. Mycoses 57: 400-405. doi: 10.1111/ myc. 12174

2. Antúnez G, Ramírez W, Rodríguez Y, García L, Flores A. 2014. Tricofitosis bovina: tratamiento preventivo y curativo. REDVET 15(4). [Internet]. Disponible en: http://www.veterinaria.org/revistas/redvet/n040414/041408.pdf

3. Antúnez G Ramírez $W$, Rodríguez, $Y$. 2012. Dermatofitosis bovina: su prevención y tratamiento. REDVET 13(3). [Internet]. Disponible en: http:// www.veterinaria.org/revistas/redvet/ n030312/031216.pdf

4. Avante M, Campos C, Ferreira M, Martins I, Souza G, Avanza M. 2009. Dermatofitose em grandes animais. Rev Cient Eletr Med Vet 7(12). [Internet]. Disponible en: http://faef.revista.inf.br/ imagens_arquivos/arquivos_destaque/ 7FYvdxr2TAJgoef_2013-6-21-10-396.pdf
5. Betancourt $O$, Salas V, Otarola A, Zaor L, Salas E, Neumann J. 2009. Microsporum canis en gatos dermatológicamente sanos en Temuco, Chile. Rev Iberoam Micol 26: 206-210.

6. Buitrago-Mejia J, Diaz-Cueto M, Suarez-Chica A, Cardona-Alvarez J. 2017. Distribución geográfica de la casuística clínica bovina del servicio ambulatorio de grandes animales de la Universidad de Córdoba (Colombia). Rev Med Vet 34: 101-113. doi: 10.19052/ mv.4259

7. Cardona J, Martínez M, Maza L. 2017. Casuística clínica más frecuente en el servicio ambulatorio de grandes animales de la Universidad de Córdoba, Colombia. Rev Colombiana Cienc Anim 9: 66-72.

8. Cardona J. 2016. Conceptos generales sobre dermatología tropical en grandes animales. Mem I Seminario Internacional de Reproducción y Clínica en Bovinos, Ovinos y Equinos. Bogotá, Colombia.

9. Chermette R, Ferreiro L, Guillot J. 2008. Dermatophytoses in animals. Mycopathologia 166: 385-405. doi: 10.1007/s11046-008-9102-7

10. Cornegliani L, Persico P, Colombo S. 2009. Canine nodular dermatophytosis (kerion): 23 cases. Vet Dermatol 20: 185190. doi: 10.1111/j.1365-3164.2009.00749.x

11. García L, López R, Ramírez R, Rodríguez, L, Nevárez, M. 2012. Descripción de un brote de dermatofitosis en bovinos en el trópico mexicano. REDVET 13(7). [Internet]. Disponible en: http://www.veterinaria.org/revistas/ redvet/n070712/071214.pdf

12. Khosravi A, Mahmoudi M. 2003. Dermatophytes isolated from domestic animals in Iran. Mycoses 46: 222-225. doi: 10.1046/j.1439-0507.2003.00868.x

13. $\operatorname{Mrad} A$. 2006. Ética en la investigación con modelos animales experimentales. Alternativas y las 3 RS de Russel. Una responsabilidad y un compromiso ético 
que nos compete a todos. Rev Colomb Bioética 1(1): 163-183.

14. Pabón J, Eslava J, Gómez, R. 2001. Generalidades de la distribución espacial y temporal de la temperatura del aire y de la precipitación en Colombia. Meteorol Colomb 4: 47-59.

15. Papini R, NardonI S, Fanelli A, Mancianti F. 2009. High infection rate of Trichophyton verrucosum in calves from Central Italy. Zoonoses Public Health 56: 59-64. doi: 10.1111/j.18632378.2008.01157.x

16. Pereira D, Meireles M. 2007. Dermatofitoses. In: Riet-Correa F (ed). Doenças de ruminantes e equídeos. Vol I. Santa Maria: Palloti. p 451-457.
17. Reis-Gomes A, Madrid I, Matos C, Telles A, Waller S, Nobre M, Meireles M. 2012. Dermatopatias fúngicas: aspectos clínicos, diagnósticos e terapêuticos. Acta Vet Brasilica 6: 272284. doi: 10.21708/avb.2012.6.4.2943

18. Rodríguez Y, Ramírez, W, Antúnez, G. 2002. Resultados de la aplicación de la acriflavina en la dermatomicosis bovina. Med Vet 19: 81-85.

19. Silveira E, Nobre M, Souza L, Faria R, Cleff M, Meireles M. 2003. Trichophyton verrucosum em bovinos com pele hígida e com lesões. Acta Sci Vet 31: 45-49. 